\title{
El proceso cIR en el mundo del modelaje en commodities: el modelo de forma reducida y de no arbitraje de dos factores de Ribeiro y Hodges (2004)
}

\author{
Carlos Armando Mejía Vega*
}

* Magíster en Finanzas. Docente-Investigador de commodities del Observatorio de Economía y Operaciones Numéricas (ODEON), Universidad Externado de Colombia.

[carlos.mejia@uexternado.edu.co].

Artículo recibido el 15 de octubre de 2017.

Aceptado el 15 de noviembre de 2017.

Para citar este artículo:

Mejía Vega, C. A. (2017). El proceso CIR en el mundo del modelaje en commodities: el modelo de forma reducida y de no arbitraje de dos factores de Ribeiro y Hodges (2004). ODEON, 13, pp. $75-98$.

DOI: https://doi.org/10.18601/17941113.n13.05 



\section{Introducción}

Una fuente inagotable de inspiración para el desarrollo de nuevos modelos de forma reducida ${ }^{1} \mathrm{y}$ de no arbitraje $^{2}$ para los mercados financieros internacionales de commodities $^{3}$ es, sin lugar a dudas, la del mundo del modelaje de las tasas de interés ${ }^{4}$. En efecto, los modelos estocásticos de tasa de interés han tenido una influencia preponderante en el desarrollo de los modelos de pricing de los contratos futuros, que pertenecen a una clase de contratos futuros sobre commodities bajo la sombrilla de la llamada Storage Theory. Algunos ejemplos famosos en este sentido son los modelos de Gibson y Schwartz (1990) y de Schwartz (1997) que adoptaron el modelo de Vasicek $(1977)^{5}$ para modelar la dinámica a través del tiempo de la tasa instantánea spot y neta de conveniencia, y los modelos de Miltersen (2003)

1 Siguiendo a Carmona y Ludkovski (2004), uno de los primeros supuestos de muchos modelos dentro del campo del modelaje de los mercados financieros internacionales de commodities es la existencia de un mercado global en el que es posible intercambiar el commodity estandarizado que hace parte de la clase de contratos futuros sobre commodities a través de contratos spot. Esta particularidad da origen a un precio spot que llamaremos precio spot estándar (Mejía, 2015). Ahora bien, a partir de este supuesto podemos clasificar los modelos en dos grupos en general. De un lado, se encuentran aquellos en tiempo discreto, conocidos como modelos estructurales, que se enfocan en las fuerzas de la oferta, la demanda y el almacenamiento del commodity en términos generales, y que buscan replicar el precio spot estándar de equilibrio a través de aproximaciones numéricas. Del otro lado, se encuentran los de tiempo continuo, conocidos como modelos de forma reducida, que son esencialmente descriptivos, y que buscan replicar (directa o indirectamente) el patrón de reversión a la media de dicha variable (Ribeiro y Hodges, 2004a).

2 Siguiendo a Kellerhals (2001), los modelos de forma reducida y de no arbitraje son aquellos que presumen que el commodity estandarizado es un activo almacenable y, por ende, se deben considerar los costos y desperdicios, así como los beneficios asociados al almacenamiento del mismo hasta su entrega, con el fin de determinar el precio futuro de un contrato futuro que pertenece a una clase de contratos futuros sobre commodities. Los beneficios generalmente se sintetizan en una tasa de interés continua conocida como tasa instantánea spot y bruta de conveniencia (Carmona y Ludkovski, 2004). El término "bruta" deriva del hecho de que existe una segunda tasa en la que se restan los costos y desperdicios (expresados también como una tasa instantánea) conocida como la tasa instantánea spot y neta de conveniencia (Law, 2009).

$3 \quad$ Siguiendo a Mejía (2015), los mercados financieros internacionales de commodities son aquellos en los que se negocian commodities a través de contratos estandarizados llamados contratos futuros.

4 En efecto, un campo de investigación dentro del modelaje de los mercados financieros internacionales de commodities corresponde a utilizar los distintos modelos estocásticos de tasa corta para modelar la dinámica tanto de la tasa instantánea spot y bruta de conveniencia, como de la tasa instantánea spot y neta de conveniencia dada la naturaleza de ambas variables.

$5 \quad$ El cual corresponde al proceso Ornstein-Uhlenbeck.

pp. $75-98 \cdot$ n. $^{\circ} 13 / 2017$ 
y de Carmona y Ludkovski (2004) que adoptan el de Hull y White (1990) para modelar esta misma variable.

En ese sentido, la influencia del modelo de los profesores John Carrington Cox, Jonathan Ingersoll y, principalmente, de Stephen Ross, que apareció en un artículo publicado en 1985 en Econometrica ${ }^{6}$, también se haría presente. En este caso, los encargados de incorporar este proceso fueron la matemática Diana Ribeiro y el matemático y profesor Stewart Hodges en un working paper publicado en el año 2004 por la Warwick Business School ${ }^{7}$, dado que optaron por trabajar con la tasa instantánea spot y bruta de conveniencia y, en ese sentido, debían evitar que dicha tasa adquiriera valores negativos para así impedir posibilidades teóricas de arbitraje $^{8}$. Dado lo anterior, y como conmemoración al profesor Stephen Ross, en el presente artículo se pretende exponer el mencionado modelo como herramienta para realizar el pricing de los contratos futuros que hacen parte de una clase de contratos futuros sobre commodities y su método general de calibración.

La estructura del artículo es la siguiente: la primera parte es la introducción; en la segunda parte se realizará una descripción general del modelo y el proceso de pricing para los contratos futuros que pertenecen a una clase de contratos futuros sobre commodities. En la tercera parte se explicará, en líneas generales, el proceso de calibración, y en la cuarta parte se presentan las conclusiones.

\section{Modelo general}

\subsection{Descripción general}

Dado un espacio de probabilidad filtrado $\left[\Omega, \mathfrak{I},\left(\mathfrak{I}_{t}\right)_{t \geq 0}, \mathbb{P}\right]$, el modelo de forma reducida y de no arbitraje de dos factores de Ribeiro y Hodges (2004) (en adelante

$6 \quad$ El título del artículo es: A theory of the term structure of interest rates.

7 El título del documento es: A two-factor model for commodity prices and futures valuation.

8 Esto no ocurre para el caso de la tasa instantánea spot y neta de conveniencia en la medida que sí puede tomar valores negativos (Gibson y Schwartz, 1990). Hay un fuerte debate en este sentido, ya que Ribeiro y Hodges (2004) señalan que una de las fallas de los modelos de forma reducida de no arbitraje de dos factores, tanto de Gibson y Schwartz (1990) como de Schwartz (1997), es que permiten oportunidades de arbitraje ya que la tasa de conveniencia puede adquirir valores negativos. Sin embargo, es preciso especificar que la variable con la que se trabaja en cada caso es diferente. Para los primeros la tasa es de carácter neto, mientras que para los segundos es de carácter bruto.

pp. $75-98 \cdot$ n. $^{\circ} 13 / 2017$ 
el modelo de Ribeiro y Hodges, 2004) establece que el precio spot estándar sigue el siguiente proceso estocástico9:

$$
d S(t)=[\mu+\mathrm{c}-\delta(t)] S(t) d t+\sigma_{1} \sqrt{\delta(t)} S(t) d W_{1}(t)
$$

Mientras que la tasa instantánea spot y bruta de conveniencia sigue un proceso Cox, Ingersoll, Ross (de aquí en adelante CIR) de la siguiente forma:

$$
d \delta(t)=k[\alpha-\delta(t)] d t+\sigma_{2} \sqrt{\delta(t)} d W_{2}(t)
$$

Y ambos siguen el siguiente proceso estocástico conjunto:

$$
d W_{1}(t) d W_{2}(t)=\rho d t
$$

donde:

$d t$ : incremento infinitesimal del tiempo.

$d S(t)$ : incremento del precio spot estándar durante el intervalo $(t, t+d t)$.

$\mu$ : componente de tendencia instantáneo del retorno total del precio spot estándar. $\sigma_{1}$ : componente de difusión instantáneo del retorno exclusivo del precio spot estándar.

9 La incorporación de la raíz de la tasa instantánea spot y bruta de conveniencia en el término de difusión del precio spot estándar la incluyen Ribeiro y Hodges (2004) por dos razones concretas (Ribeiro y Hodges, 2004b):

- Desde un punto de vista teórico, la volatilidad del precio spot estándar es heteroscedástica y refleja las condiciones de la oferta, la demanda y los inventarios del commodity en general. En particular, dicha volatilidad se incrementa con los grados de backwardation. Esto es, entre más alta sea la condición de backwardation, más alta será la de la tasa de conveniencia. De esta forma, tasas de conveniencia altas implican bajos niveles de inventario $\mathrm{y}$, por ende, la volatilidad del precio spot estándar se encontraría relacionada positivamente con el valor de la tasa de conveniencia.

- Desde un punto de vista matemático, para hallar una solución cerrada se requiere de la presencia de dicha raíz en el componente de difusión, ya que, de lo contrario, al momento de resolver la ecuación diferencial parcial, una de las ecuaciones diferenciales ordinarias es increíblemente compleja. 
- $d W_{1}(t)$ : incremento, durante el intervalo $(t, t+d t)$, de un movimiento Browniano estándar bajo $\mathbb{P}$, asociado al precio spot estándar, con valor esperado 0 y varianza $t$.

- $\quad d \delta(t)$ : incremento de la tasa instantánea spot y bruta de conveniencia durante el intervalo $(t, t+d t)$.

- $\alpha$ : valor esperado de largo plazo de la tasa instantánea spot y bruta de conveniencia.

- $\quad k>0$ : velocidad de reversión de la tasa instantánea spot y bruta de conveniencia en torno a su valor esperado de largo plazo.

- $\sigma_{2}$ : componente de difusión de la tasa instantánea spot y bruta de conveniencia.

- $d W_{2}(t)$ : incremento durante el intervalo $(t, t+d t)$, de un movimiento Browniano estándar bajo $\mathbb{P}$, asociado a la tasa instantánea spot y bruta de conveniencia con valor esperado 0 y varianza $t$.

- $\rho$ : coeficiente de correlación entre el retorno instantáneo del precio spot estándar y la tasa instantánea spot y bruta de conveniencia.

\subsection{Proceso de pricing}

Dado que el modelo de Ribeiro y Hodges (2004) es un modelo de forma reducida y de no arbitraje, entonces el precio futuro en el momento de tiempo $t$ de un contrato futuro que pertenece a una clase de contratos futuros sobre commodities con vencimiento en $T_{j}$, tales que $0 \leq t \leq T_{j}$ y donde $j=1,2 ; \ldots, J$, siendo $J$ el vencimiento líquido más lejano a lo largo de la curva forward $F\left(t ; T_{j}\right)$, se define como una función de las variables de estado bajo (en este caso el precio spot estándar y la tasa instantánea spot y bruta de conveniencia) y del tiempo al vencimiento bajo la medida martingala-equivalente o riesgo-neutral $\mathbb{Q}^{*}$ :

$$
F\left(t ; T_{j}\right)=f_{\mathbb{Q}^{*}}\left[S(t), \delta(t), T_{j}-t\right]
$$

Dado lo anterior, se requiere primero transformar el modelo de Ribeiro y Hodges (2004) bajo la medida de probabilidad riesgo-neutral o martingala-equivalente. 


\subsubsection{Modelo de Ribeiro y Hodges (2004) riesgo-neutral}

Bajo un espacio de probabilidad filtrado $\left[\Omega, \Im,\left(\Im_{t}\right)_{t \geq 0}, \mathbb{P}\right]$ y expresando $W(t)=\left[W_{1}(t), W_{2}(t)\right]$ como los dos movimientos Brownianos estándar bajo $\mathbb{P}$, se propone el siguiente proceso adaptado:

$$
\theta(t)=\left[\theta_{1}(t), \theta_{2}(t)\right]
$$

donde:

$\theta_{1}(t)$ : precio de mercado del riesgo asociado al precio spot estándar. $\theta_{2}(t)$ : precio de mercado del riesgo asociado a la tasa instantánea bruta y spot de conveniencia.

Bajo este esquema, el riesgo de mercado asociado al precio spot estándar se puede cubrir usando la clase de contratos futuros sobre commodities que emplea precisamente el commodity estandarizado como su subyacente. Con base en esto, la prima de riesgo de mercado quedaría completamente especificada y podríamos definir $\theta_{1}$ de la siguiente forma:

$$
\theta_{1}(t)=\frac{\mu-r}{\sigma_{1} \sqrt{\delta(t)}}
$$

Donde $r$ es la tasa instantánea libre de riesgo que se asume constante ${ }^{10}$.

Sin embargo, no existe un instrumento que se pueda utilizar para cubrir el riesgo de mercado asociado a la tasa instantánea bruta y spot de conveniencia. Con base en esto, la prima de riesgo de mercado no puede especificarse, y debe incluirse de manera explícita en $\theta_{2}(t)$ de la siguiente manera:

$$
\theta_{2}(t)=\frac{\lambda}{\sigma_{2} \sqrt{\delta(t)}}
$$

10 Un trabajo interesante por desarrollar en este sentido sería el de dotar de naturaleza estocástica a dichas tasas de interés dentro del modelo de Ribeiro y Hodges (2004), de la misma forma que Schwartz (1997) lo hizo con su modelo de forma reducida y no de arbitraje de dos factores. 
Donde $\lambda$ es la prima de riesgo de mercado que se asume constante ${ }^{11}$.

Teniendo en cuenta (4) y (5), podemos expresar (3) también como:

$$
\theta(t)=\left[\theta_{1}(t), \theta_{2}(t)\right]=\left(\frac{\mu-r}{\sigma_{1} \sqrt{\delta(t)}}, \frac{\lambda}{\sigma_{2} \sqrt{\delta(t)}}\right)
$$

Dado que $\theta(t)$ satisface la condición de Novikov bajo $\mathbb{P}$ (Ribeiro y Hodges, 2004a), entonces existe una medida de probabilidad equivalente (que es riesgo-neutral o martingala-equivalente dada la forma como se definió (6)), tal que (Girsanov, 1960):

$$
\tilde{W}(t)=\left[W_{1}(t)+\int_{0}^{t}\left(\frac{\mu-r}{\sigma_{1} \sqrt{\delta(u)}}\right) d u, W_{2}(t)+\int_{0}^{t}\left(\frac{\lambda}{\sigma_{2} \sqrt{\delta(u)}}\right) d u\right]
$$

Donde $\tilde{W}(t)$ es un movimiento Browniano estándar bajo la medida riesgo-neutral o martingala-equivalente $\mathbb{Q}^{*}$. La forma diferencial de (7) es la siguiente:

$$
\begin{aligned}
& d \tilde{W}_{1}(t)=d W_{1}(t)+\left(\frac{\mu-r}{\sigma_{1} \sqrt{\delta(t)}}\right) d t \\
& d \tilde{W}_{2}(t)=d W_{2}(t)+\left(\frac{\lambda}{\sigma_{2} \sqrt{\delta(t)}}\right) d t
\end{aligned}
$$

O de manera alternativa:

$$
d W_{1}(t)=d \tilde{W}_{1}(t)-\left(\frac{\mu-r}{\sigma_{1} \sqrt{\delta(t)}}\right) d t
$$

11 Un trabajo interesante por desarrollar en este sentido sería el de dotar de naturaleza estocástica a dichas primas en el modelo de Ribeiro y Hodges (2004), de la misma forma que Carmona y Ludkovski (2004) lo hicieron con el modelo de forma reducida y de no de arbitraje de dos factores de Schwartz (1997). 


$$
d W_{2}(t)=d \tilde{W}_{2}(t)-\left(\frac{\lambda}{\sigma_{2} \sqrt{\delta(t)}}\right) d t
$$

Finalmente, reemplazamos (9a) en (1a) y (9b) en (1b) para obtener el modelo de Ribeiro y Hodges (2004) riesgo-neutral ${ }^{12}$ :

$$
\begin{gathered}
d S(t)=[r+c-\delta(t)] S(t) d t+\sigma_{1} \sqrt{\delta(t)} S(t) d \tilde{W}_{1}(t) \\
d \delta(t)=\{k[\alpha-\delta(t)]-\lambda\} d t+\sigma_{2} \sqrt{\delta(t)} d \tilde{W}_{2}(t) \\
d \tilde{W}_{1}(t) d \tilde{W}_{2}(t)=\rho d t
\end{gathered}
$$

Donde $\tilde{W}(t)=\left[\tilde{W}_{1}(t), \tilde{W}_{2}(t)\right]$ son dos movimientos Brownianos estándar bajo $\mathbb{Q}^{*}$.

\subsubsection{Solución cerrada}

Dado el modelo de Ribeiro y Hodges (2004) riesgo-neutral de (10), y retomando (2), se debe cumplir con la siguiente ecuación diferencial parcial al aplicar el lema de Itô y el principio de no arbitraje ${ }^{13}$ :

$$
\begin{gathered}
{[(r+c)-\delta(t)]-S(t)\left\{\frac{\partial F\left[t, S(t), \delta(t) ; T_{j}\right]}{\partial \delta(t)}\right\}} \\
+\{k[\alpha-\delta(t)]-\lambda\}\left\{\frac{\partial F\left[t, S(t), \delta(t) ; T_{j}\right]}{\partial \delta(t)}\right\} \\
+\frac{1}{2} \sigma_{1}^{2} \delta(t) S(t)^{2}\left\{\frac{\partial^{2} F\left[t, S(t), \delta(t) ; T_{j}\right]}{\partial S(t)^{2}}\right\}
\end{gathered}
$$

12 Véase el anexo A.

13 Véase el anexo B. 


$$
\begin{gathered}
+\frac{1}{2} \sigma_{2}^{2} \delta(t)\left\{\frac{\partial^{2} F\left[t, S(t), \delta(t) ; T_{j}\right]}{\partial \delta(t)^{2}}\right\} \\
+\rho \sigma_{1} \sigma_{2} S(t) \delta(t)\left\{\frac{\partial^{2} F\left[t, S(t), \delta(t) ; T_{j}\right]}{\partial S(t) \partial \delta(t)}\right\} \\
-\frac{\partial F\left[t, S(t), \delta(t) ; T_{j}\right]}{\partial T_{j}}=0
\end{gathered}
$$

Sujeto a la condición de frontera $F\left[T_{j}, S\left(T_{j}\right), \delta\left(T_{j}\right) ; T_{j}\right]=S\left(T_{j}\right)$. Siguiendo a Ribeiro y Hodges (2004b), la solución de (11) es la siguiente ${ }^{14}$ :

$$
\operatorname{Ln}\left\{F\left[t, S(t), \delta(t), T_{j}\right]\right\}=\operatorname{Ln}[S(t)]+A\left(T_{j}-t\right)-\delta(t) B\left(T_{j}-t\right)
$$

Donde:

$$
\begin{gathered}
A\left(T_{j}-t\right)=(r+c)\left(T_{j}-t\right)+(\lambda-k \alpha) \int_{t}^{T_{j}} B(u) d u \\
\int_{t}^{T_{j}} B(u) d u=\frac{2}{k_{1}\left(k_{1}+k_{2}\right)} \operatorname{Ln}\left[\frac{\left(k_{1}+k_{2}\right) e^{k_{1}\left(T_{j}-t\right)}+k_{1}-k_{2}}{2 k_{1}}\right] \\
+\frac{2}{k_{1}\left(k_{1}-k_{2}\right)} \operatorname{Ln}\left[\frac{k_{1}+k_{2}+\left(k_{1}-k_{2}\right) e^{-k_{1}\left(T_{j}-t\right)}}{2 k_{1}}\right]
\end{gathered}
$$




$$
\begin{gathered}
B\left(T_{j}-t\right)=\frac{2\left[1-e^{-k_{1}\left(T_{j}-t\right)}\right]}{k_{1}+k_{2}+\left(k_{1}-k_{2}\right) e^{-k_{1}\left(T_{j}-t\right)}} \\
k_{1}=\sqrt{k_{2}^{2}+2 \sigma_{2}^{2}} \\
k_{2}=\alpha-\rho \sigma_{1} \sigma_{2}
\end{gathered}
$$

\section{Calibración}

\subsection{Proceso general}

Dado que las variables de estado en este tipo de modelos (precio spot estándar y tasa instantánea spot y bruta de conveniencia) no son visibles para muchos commodities en general, entonces uno de los mejores esquemas para calibrar el modelo es a través del filtro de Kalman y el método de Máxima Log-Verosimilitud. Sin embargo, es pertinente indicar que dado que el proceso CIR no sigue una distribución normal sino una distribución chi-cuadrada no centrada (Cox, Ingersoll y Ross, 1985), entonces los parámetros estimados son cuasi-óptimos y no óptimos. Para obtener la ecuación de transición se empleará el mismo método propuesto por Ribeiro y Hodges (2004b):

- Se emplea el método de Euler-Maruyama para (1a). Sin embargo, como esta primera aproximación puede producir valores negativos de $S(t)$, se evita esta dificultad escribiendo $f[t, S(t)]=\operatorname{Ln}[S(t)]$ y empleando el método de Euler-Maruyama para $\operatorname{Ln}[S(t)]$.

- Se discretiza la solución numérica exacta de (1b).

- Se incorpora de manera indirecta el proceso estocástico conjunto (1c) dentro de la matriz de covarianza de la distribución normal multivariada de las perturbaciones seriales no correlacionadas empleada durante el proceso de discretización, a través de la fórmula del coeficiente de correlación de Pearson. 


\subsection{El estado de espacio de forma para el modelo de Ribeiro y Hodges (2004)}

Como se indicó previamente, esta aproximación consiste en aplicar el método de Euler-Maruyama para $\operatorname{Ln}[S(t)]$. Con base en esto, se debe obtener la ecuación diferencial para $\operatorname{Ln}[S(t)]$. Considerando que (1a) es un proceso de Itô, se define la función $f[t, S(t)]=\operatorname{Ln}[S(t)]$ y se aplica el lema de Itô (Ribeiro y Hodges, 2004b):

$$
d\{L n[S(t)]\}=\left[\mu+c-\left(1+\frac{\sigma_{1}^{2}}{2}\right) \delta(t)\right] d t+\sigma_{1} \sqrt{\delta(t)} d W_{1}(t)
$$

Con base en lo anterior, (13), (1.1b) y (1.1c) se utilizarán para construir la ecuación de transición, mientras que (12) se utilizará para la ecuación de medida.

2.2.1 Los vectores de estado y de medida para el modelo de Ribeiro y Hodges (2004)

En el caso concreto del modelo de Ribeiro y Hodges (2004) las variables que no son observables, para cada momento del tiempo $t$, son el logaritmo natural del precio spot estándar y la tasa instantánea bruta y spot de conveniencia. Con base en esto podemos definir el vector de estado de la siguiente forma:

$$
x_{t}=\left[\begin{array}{c}
\operatorname{Ln}\left(S_{t}\right) \\
\delta_{t}
\end{array}\right]
$$

donde:

- $x_{t}$ : vector estado de $2 \times 1$.

- $\operatorname{Ln}\left(S_{t}\right)$ : logaritmo natural del precio spot estándar en el momento del tiempo $t$.

- $\delta_{t}$ : tasa instantánea spot y bruta de conveniencia en el momento de tiempo $t$. 
Por otro lado, las variables observadas en cada momento del tiempo $t$ son los logaritmos naturales de los precios futuros de los J contratos futuros de la misma clase de contratos futuros sobre commodities. Con base en esto podemos definir el vector de medida de la siguiente forma:

$$
y_{t}=\left[\begin{array}{c}
\operatorname{Ln}\left(F_{t, T_{1}}\right) \\
\vdots \\
\operatorname{Ln}\left(F_{t, T_{N}}\right)
\end{array}\right]
$$

donde:

$y_{t}$ : vector de medida de $J=N \times 1$.

$\operatorname{Ln}\left(F_{t, T_{j}}\right)$ : logaritmo natural del precio futuro de una clase de contratos futuros sobre commodities con vencimiento en $T_{j}$, donde $j=1, \ldots, J=N$.

2.2.2 La ecuación de medida para el modelo de Ribeiro y Hodges (2004)

Para construir la ecuación de medida se debe discretizar (12) de la siguiente forma:

$$
y_{t}=Z_{t} x_{t}+d_{t}+\varepsilon_{t}, t=1, \ldots, N O B S
$$

donde:

$d_{t}=\left[A\left(\tau_{j, t}\right)\right]:$ vector de $N \times 1$ y $A\left(\tau_{j, t}\right)$ está dado por (12b), (12c), (12e), (11f) en tiempo discreto.

$$
Z_{t}=\left[1, B\left(\tau_{j, t}\right)\right]: \text { matriz de } N \times 2 \text { y } B\left(\tau_{j, t}\right) \text { está dado por (12d), (12e), }
$$
en tiempo discreto.

$\varepsilon_{t}$ : vector de $N \times 1$ de perturbaciones seriales no correlacionadas que siguen una distribución normal multivariada con:

$$
E\left(\varepsilon_{t}\right)=\left[\begin{array}{c}
0 \\
\vdots \\
0
\end{array}\right]_{N=J x 1}
$$




$$
\operatorname{Cov}\left(\varepsilon_{t}\right)=E\left\{\left[\varepsilon_{t}-E\left(\varepsilon_{t}\right)\right]\left[\varepsilon_{t}-E\left(\varepsilon_{t}\right)\right]^{\prime}\right\}=E\left(\varepsilon_{t} \varepsilon_{t}^{\prime}\right)=H_{t}
$$

Este vector se introduce generalmente para representar posibles errores en la información.

\subsubsection{La ecuación de transición para el modelo de Ribeiro y Hodges (2004)}

Paso 1. Método de Euler-Maruyama para el logaritmo natural del precio spot estándar

Podemos aproximar la solución numérica a (13) y discretizarla en una partición con pasos constantes $\Delta t$ a través del método de Euler-Maruyama de la siguiente forma (Platen y Bruti-Liberati, 2007):

$$
\operatorname{Ln}\left(S_{t}\right)=\operatorname{Ln}\left(S_{t-1}\right)+\left[\mu+c-\left(1+\frac{\sigma_{1}^{2}}{2}\right) \delta_{t}\right] \Delta t+\sigma_{1} \sqrt{\delta_{t}} \sqrt{\Delta t} \eta_{1, t}
$$

Donde $\eta_{1, t}$ es una variable aleatoria serial no correlacionada que sigue una distribución normal con valor esperado cero y varianza $\sigma_{1}^{2} \delta_{t} \Delta t$. Una segunda forma de expresar lo anterior es:

$$
\operatorname{Ln}\left(S_{t}\right)=\operatorname{Ln}\left(S_{t-1}\right)+\left[\mu+c-\left(1+\frac{\sigma_{1}^{2}}{2}\right) \delta_{t}\right] \Delta t+\eta_{1, t}
$$

Donde ahora $\eta_{1, t}$ es una variable aleatoria serial no correlacionada que sigue una distribución normal con valor esperado cero y varianza $\sigma_{1}^{2} \delta_{t} \Delta t$. Finalmente, podemos expresar (17) de esta otra manera para adaptarla a la ecuación de transición final:

$$
\operatorname{Ln}\left(S_{t}\right)=(\mu+c) \Delta t+\operatorname{Ln}\left(S_{t-1}\right)-\left(1+\frac{\sigma_{1}^{2}}{2}\right) \delta_{t}+\eta_{1, t}
$$

Paso 2. Discretización de la solución numérica exacta de la tasa instantánea spot y bruta de conveniencia. 
Por otro lado, necesitamos obtener la solución numérica exacta de (1.1b). Teniendo en cuenta que (1.1b) es un proceso de Itô, definimos la función $f[t, \delta(t)]=\delta(t) e^{k t}$ y aplicamos el lema de Itô:

$$
d \delta(t)=k \alpha e^{k t} d t+\sigma_{2} \sqrt{\delta(t)} e^{k t} d W_{2}(t)
$$

Para obtener la solución numérica integramos a ambos lados de (19) desde $s$ to $t$, donde $0 \leq s<t$ :

$$
\delta(t)=\delta(s) e^{-k(t-s)}+\alpha\left[1-e^{-k(t-s)}\right]+\int_{s}^{t} \sigma_{2} \sqrt{\delta(u)} e^{-k(t-u)} d W_{2}(u)
$$

A partir de (20a) podemos calcular el valor esperado y la varianza del proceso condicional a la información $\left(\mathfrak{I}_{s}\right)_{t>s \geq 0}$ :

$$
\begin{gathered}
E\left[\delta(t) \mid \mathfrak{I}_{s}\right]=\delta(s) e^{-k(t-s)}+\alpha\left[1-e^{-k(t-s)}\right] \\
\operatorname{Var}\left[\delta(t) \mid \mathfrak{I}_{s}\right]=\alpha\left(\frac{\sigma_{2}^{2}}{2 k}\right)\left[1-e^{-2 k(t-s)}\right]+\delta(s) \frac{\sigma_{2}^{2}}{k}\left[e^{-k(t-s)}-e^{-2 k(t-s)}\right]
\end{gathered}
$$

A partir de (20) podemos concluir que el proceso sigue una distribución chicuadrada no centrada condicional a la información $\mathfrak{I}_{s}$ con valor esperado de $\delta(s) e^{-k(t-s)}+\alpha\left[1-e^{-k(t-s)}\right]$ y varianza de $\alpha\left(\frac{\sigma_{2}^{2}}{2 k}\right)\left[1-e^{-2 k(t-s)}\right]+\delta(s) \frac{\sigma_{2}^{2}}{k}\left[e^{-k(t-s)}\right.$ $\left.-e^{-2 k(t-s)}\right]$. Dada la disponibilidad de un set de información $\mathfrak{I}_{t-1}$ se procede a discretizar el proceso en un contexto normal:

$$
\delta_{t}=\delta_{t-1} e^{-k \Delta t}+\alpha\left(1-e^{-k \Delta t}\right)+\eta_{2, t}
$$

Donde $\eta_{2, t}$ es una variable aleatoria serial no correlacionada que sigue una distribución normal con valor esperado cero y varianza:

$$
\operatorname{Var}\left(\eta_{2, t} \mid \mathfrak{I}_{t-1}\right)=\alpha\left(\frac{\sigma_{2}^{2}}{2 k}\right)\left(1-e^{-2 k \Delta t}\right)+\delta(s) \frac{\sigma_{2}^{2}}{k}\left(e^{-k \Delta t}-e^{-2 k \Delta t}\right)
$$


Paso 3. Construcción de la ecuación de transición e incorporación del proceso estocástico conjunto

Con base en (18) y (21) podemos incorporar el proceso estocástico conjunto en (1.1c) señalando que tanto $\eta_{1, t}$ como $\eta_{2, t}$ tienen la siguiente covarianza, obtenida mediante la aplicación de la fórmula del coeficiente de correlación de Pearson:

$$
\operatorname{Cov}\left(\eta_{1, t}, \eta_{2, t}\right)=\rho \sigma_{1} \sqrt{\delta_{t-1}} \sqrt{\Delta t} \sqrt{\operatorname{Var}\left(\eta_{2, t} \mid \mathfrak{I}_{t-1}\right)}
$$

Y podemos proponer la siguiente ecuación de transición:

$$
x_{t}=\mathbf{T} x_{t-1}+c+\eta_{t}, t=1, \ldots, N O B S
$$

donde:

$$
\begin{aligned}
& \mathbf{T}=\left[\begin{array}{cc}
1 & -\left(1+\frac{\sigma_{1}^{2}}{2}\right) \\
0 & e^{-k(t-s)}
\end{array}\right]: \text { es una matriz de } 2 \times 2 . \\
& c=\left[\begin{array}{c}
\mu+c \\
\alpha\left(1-e^{-k \Delta t}\right)
\end{array}\right]: \text { es un vector de } 2 \times 1 .
\end{aligned}
$$

$\eta_{t}$ : es un vector de $2 \times 1$ de perturbaciones seriales no correlacionadas que siguen una distribución normal multivariada con:

$$
\begin{gathered}
E\left(\eta_{t}\right)=\left[\begin{array}{c}
0 \\
0
\end{array}\right] \\
\operatorname{Cov}\left(\eta_{t}\right)=E\left\{\left[\eta_{t}-E\left(\eta_{t}\right)\right]\left[\eta_{t}-E\left(\eta_{t}\right)\right]\right\}=E\left(\eta_{t} \eta_{t}^{\prime}\right)=Q \\
=\left[\begin{array}{cc}
\sigma_{1}^{2} \delta_{t} \Delta t & \rho \sigma_{1} \sqrt{\delta_{t-1}} \sqrt{\Delta t} \sqrt{\operatorname{Var}\left(\eta_{2, t} \mid \mathfrak{I}_{t-1}\right)} \\
\rho \sigma_{1} \sqrt{\delta_{t-1}} \sqrt{\Delta t} \sqrt{\operatorname{Var}\left(\eta_{2, t} \mid \mathfrak{I}_{t-1}\right)} & \operatorname{Var}\left(\eta_{2, t} \mid \mathfrak{I}_{t-1}\right)
\end{array}\right]
\end{gathered}
$$


Una vez se cuenta con las ecuaciones de transición y de medida, el proceso se puede calibrar siguiendo la metodología del filtro de Kalman y el método de Máxima Log-Verosimilitud estándar dada la información de una clase de contratos futuros sobre commodities ${ }^{15}$.

\section{Conclusiones}

El recuento del modelo de Ribeiro y Hodges (2004) es una excelente forma de conmemorar al profesor Stephen Ross, ya que sin el proceso CIR no se hubieran podido extender los trabajos de Gibson y Schwartz (1990) y de Schwartz (1997) como ellos lo hicieron. Modelar la tasa instantánea spot y bruta de conveniencia es reconocida hoy como una de las formas más comunes de implementar el proceso CIR al mundo del modelaje en commodities.

Sin embargo, es fundamental reconocer que la calibración de este modelo a través del filtro de Kalman puede llegar a ser problemática. Lo anterior debido a que, si bien la relación entre los precios futuros y las variables de estado es lineal, el ruido no es gaussiano sino chi-cuadrado no centrado. De esta forma, los parámetros obtenidos son cuasi-óptimos y no óptimos. Se abre entonces la pregunta de cómo estimar los parámetros siguiendo esta metodología, pero con ruidos no gaussianos. A este respecto, si bien la literatura propone varios acercamientos desde disciplinas como las matemáticas, lo cierto es que desde el campo del modelaje en commodities el tema aún es precario y abre bastantes puertas para su estudio en el futuro.

\section{Referencias}

Carmona, R. y Ludkovski, M. (2004). Spot convenience yield models for the energy markets. Contemporary Mathematics, 351, 65-80.

\footnotetext{
15 Para este año 2018 se espera la publicación de un libro en el que se explica la forma de calibrar, a través del filtro de Kalman y el método de máxima log-verosimilitud, el modelo de Schwartz (1997) de forma reducida y de no arbitraje de dos factores titulado: Parameter calibration of the Schwartz (1997) reduced-form, no-arbitrage Two-Factor model through the expectation maximization algorithm or prediction error decomposition. Este libro puede servir de referencia para entender la lógica de calibración del modelo de Ribeiro y Hodges una vez se construyen las ecuaciones de transición y de medida para este, ya que la metodología es la misma.
}

pp. $75-98 \cdot$ n. $^{\circ} 13 / 2017$ 
Cox, J. C., Ingersoll, J. E. y Ross, S. A. (1985). A theory of the term structure of interest rates. Econometrica, 53 (2), 385-408.

Gibson, R. y Schwartz, E. S. (1990). Stochastic convenience yield and the pricing of oil contingent claims. The Journal of Finance, 45 (3), 959-976.

Girsanov, I. V. (1960). On transforming a certain class of stochastic processes by absolutely continuous substitution of measures. Theory of Probability \& Its Applications, 5 (3), 285-301.

Hull, J. y White, A. (1990). Pricing interest-rate-derivative securities. The Review of Financial Studies, 3 (4), 573-592.

Kellerhals, B. P. (2001). Financial Pricing Models in Continuous Time and Kalman Filtering. Berlin: Springer-Verlag Berlin Heidelberg.

Law, H. S. (2009). On the modelling, design and valuation of commodity derivatives. Doctoral dissertation, The University of Manchester.

Mejía, C. A. (2015). Una introducción general a los mercados de commodities a nivel internacional. Recuperado de https://papers.ssrn.com/sol3/papers.cfm?abstract_ id $=3062233$

Miltersen, K. R. (2003). Commodity price modelling that matches current observables: A new approach. Quantitative Finance, 3 (issue 1), 51-58.

Platen, E. y Bruti-Liberati, N. (2007). Numerical Solution of Stochastic Differential Equations with Jumps inf Finance. Sidney: Springer.

Ribeiro, D. R. y Hodges, S. D. (2004a). Equilibrium Model for Commodity Prices: Competitive and Monopolistic Markets. Recuperado de https:/warwick.ac.uk/fac/soc/ wbs/subjects/finance/research/wpaperseries/pp_04.130.pdf

Ribeiro, D. R. y Hodges, S. D. (2004b). A two-factor model for commodity prices and futures valuation. Recuperado de https://pdfs.semanticscholar.org/e395/228d9a0e5 1a302772cd50ef76108204e8ff8.pdf

Schwartz, E. S. (1997). The stochastic behavior of commodity prices: Implications for valuation and hedging. The Journal of Finance, 52 (3), 923-973. 
Vasicek, O. (1977). An equilibrium characterization of the term structure. Journal of Financial Economics, 5 (2), 177-188.

Anexo A. Transformación del modelo de Ribeiro y Hodges (2004) al modelo de Ribeiro y Hodges (2004) riesgo-neutral

Reemplazamos (9a) en (1a):

$$
\begin{gathered}
d S(t)=[\mu+\mathrm{c}-\delta(t)] S(t) d t+\sigma_{1} \sqrt{\delta(t)} S(t)\left[d \tilde{W}_{1}(t)-\left(\frac{\mu-r}{\sigma_{1} \sqrt{\delta(t)}}\right) d t\right] \\
d S(t)=[\mu+\mathrm{c}-\delta(t)] S(t) d t+\sigma_{1} \sqrt{\delta(t)} S(t) d \tilde{W}_{1}(t)-\sigma_{1} \sqrt{\delta(t)} S(t)\left(\frac{\mu-r}{\sigma_{1} \sqrt{\delta(t)}}\right) d t \\
d S(t)=[\mu+\mathrm{c}-\delta(t)] S(t) d t+\sigma_{1} \sqrt{\delta(t)} S(t) d \tilde{W}_{1}(t)-\mu S(t) d t+r S(t) d t \\
d S(t)=[\mu+\mathrm{c}-\delta(t)] S(t) d t-\mu S(t) d t+r S(t) d t+\sigma_{1} \sqrt{\delta(t)} S(t) d \tilde{W}_{1}(t) \\
d S(t)=[\mathrm{c}-\delta(t)] S(t) d t+r S(t) d t+\sigma_{1} \sqrt{\delta(t)} S(t) d \tilde{W}_{1}(t) \\
d S(t)=[r+\mathrm{c}-\delta(t)] S(t) d t+\sigma_{1} \sqrt{\delta(t)} S(t) d \tilde{W}_{1}(t)
\end{gathered}
$$

Reemplazamos (9b) en (1b):

$$
\begin{gathered}
d \delta(t)=k[\alpha-\delta(t)] d t+\sigma_{2} \sqrt{\delta(t)}\left[d \tilde{W}_{2}(t)-\left(\frac{\lambda}{\sigma_{2} \sqrt{\delta(t)}}\right) d t\right] \\
d \delta(t)=k[\alpha-\delta(t)] d t+\sigma_{2} \sqrt{\delta(t)} d \tilde{W}_{2}(t) d t-\sigma_{2} \sqrt{\delta(t)}\left(\frac{\lambda}{\sigma_{2} \sqrt{\delta(t)}}\right) d t
\end{gathered}
$$




$$
\begin{aligned}
& d \delta(t)=k[\alpha-\delta(t)] d t+\sigma_{2} \sqrt{\delta(t)} d \tilde{W}_{2}(t) d t-\lambda d t \\
& d \delta(t)=k[\alpha-\delta(t)] d t-\lambda d t+\sigma_{2} \sqrt{\delta(t)} d \tilde{W}_{2}(t) d t \\
& d \delta(t)=\{k[\alpha-\delta(t)]-\lambda\} d t+\sigma_{2} \sqrt{\delta(t)} d \tilde{W}_{2}(t) d t \\
& d \delta(t)=[k \alpha-k \delta(t)-\lambda] d t+\sigma_{2} \sqrt{\delta(t)} d \tilde{W}_{2}(t) d t \\
& d \delta(t)=\{k[\alpha-\delta(t)]-\lambda\} d t+\sigma_{2} \sqrt{\delta(t)} d \tilde{W}_{2}(t)
\end{aligned}
$$

\section{Anexo B. Ecuación diferencial parcial}

Dado que (10a) y (10b) son ambos procesos de Itô y considerando (2), entonces podemos aplicar el lema de Itô en su forma multivariada:

$$
\begin{gathered}
d\left\{F\left[t, S(t), \delta(t) ; T_{j}\right]\right\} \\
=<\frac{\partial F\left[t, S(t), \delta(t) ; T_{j}\right]}{\partial t}+[r+c-\delta(t)] S(t)\left\{\frac{\partial F\left[t, S(t), \delta(t) ; T_{j}\right]}{\partial S(t)}\right\} \\
+\{k[\alpha-\delta(t)]-\lambda\}\left\{\frac{\partial F\left[t, S(t), \delta(t) ; T_{j}\right]}{\partial \delta(t)}\right\} \\
+\frac{1}{2}\left[\sigma_{1} \sqrt{\delta(t)} S(t)\right]^{2}\left\{\frac{\partial^{2} F\left[t, S(t), \delta(t) ; T_{j}\right]}{\partial S(t)^{2}}\right\} \\
+\frac{1}{2}\left[\sigma_{2} \sqrt{\delta(t)}\right]^{2}\left\{\frac{\partial^{2} F\left[t, S(t), \delta(t) ; T_{j}\right]}{\partial \delta(t)^{2}}\right\}
\end{gathered}
$$




$$
\begin{gathered}
+\rho \sigma_{1} \sigma_{2} \delta(t) S(t)\left\{\frac{\partial^{2} F\left[t, S(t), \delta(t) ; T_{j}\right]}{\partial S(t) \partial \delta(t)}\right\}>d t \\
+\sigma_{1} \sqrt{\delta(t)} S(t) \frac{\partial F\left[t, S(t), \delta(t) ; T_{j}\right]}{\partial S(t)} d \tilde{W}_{1}(t) \\
+\sigma_{2} \sqrt{\delta(t)} \frac{\partial F\left[t, S(t), \delta(t) ; T_{j}\right]}{\partial \delta(t)} d \tilde{W}_{2}(t)
\end{gathered}
$$

Al aplicar el principio de no arbitraje y recordar que $\frac{\partial F\left[t, S(t), \delta(t) ; T_{j}\right]}{\partial t}=$ $-\frac{\partial F\left[t, S(t), \delta(t) ; T_{j}\right]}{\partial\left(T_{j}-t\right)}$, podemos expresar la ecuación anterior como la siguiente ecuación diferencial parcial:

$$
\begin{gathered}
{[(r+c)-\delta(t)] S(t)\left\{\frac{\partial F\left[t, S(t), \delta(t) ; T_{j}\right]}{\partial S(t)}\right\}} \\
+\{k[\alpha-\delta(t)]-\lambda\}\left\{\frac{\partial F\left[t, S(t), \delta(t) ; T_{j}\right]}{\partial \delta(t)}\right\} \\
+\frac{1}{2} \sigma_{1}^{2} \delta(t) S(t)^{2}\left\{\frac{\partial^{2} F\left[t, S(t), \delta(t) ; T_{j}\right]}{\partial S(t)^{2}}\right\} \\
+\frac{1}{2} \sigma_{2}^{2} \delta(t)\left\{\frac{\partial^{2} F\left[t, S(t), \delta(t) ; T_{j}\right]}{\partial \delta(t)^{2}}\right\}
\end{gathered}
$$




$$
+\rho \sigma_{1} \sigma_{2} S(t) \delta(t)\left\{\frac{\partial^{2} F\left[t, S(t), \delta(t) ; T_{j}\right]}{\partial S(t) \partial \delta(t)}\right\}-\frac{\partial F\left[t, S(t), \delta(t) ; T_{j}\right]}{\partial T_{j}}=0
$$

\section{Anexo C. Solución de la ecuación diferencial parcial}

Dada la ecuación diferencial parcial:

$$
\begin{gathered}
{[(r+c)-\delta(t)] S(t)\left\{\frac{\partial F\left[t, S(t), \delta(t) ; T_{j}\right]}{\partial S(t)}\right\}} \\
+\{k[\alpha-\delta(t)]-\lambda\}\left\{\frac{\partial F\left[t, S(t), \delta(t) ; T_{j}\right]}{\partial \delta(t)}\right\} \\
+\frac{1}{2} \sigma_{1}^{2} \delta(t) S(t)^{2}\left\{\frac{\partial^{2} F\left[t, S(t), \delta(t) ; T_{j}\right]}{\partial S(t)^{2}}\right\} \\
+\frac{1}{2} \sigma_{2}^{2} \delta(t)\left\{\frac{\partial^{2} F\left[t, S(t), \delta(t) ; T_{j}\right]}{\partial \delta(t)^{2}}\right\} \\
+\rho \sigma_{1} \sigma_{2} S(t) \delta(t)\left\{\frac{\partial^{2} F\left[t, S(t), \delta(t) ; T_{j}\right]}{\partial S(t) \partial \delta(t)}\right\} \frac{\partial F\left[t, S(t), \delta(t) ; T_{j}\right]}{\partial T_{j}}=0
\end{gathered}
$$

Asumimos una solución del tipo:

$$
\operatorname{Ln}\left\{F\left[S(t), \delta(t), T_{j}-t\right]\right\}=\operatorname{Ln}[S(t)]+B\left(T_{j}-t\right) \delta(t)+A\left(T_{j}-t\right)
$$


Donde $B\left(T_{j}-T_{j}\right)=B(0)=0$ y $A\left(T_{j}-T_{j}\right)=A(0)=0$, tales que cuando $t=T_{j}$, entonces $\operatorname{Ln}\left\{F\left[T_{j}, S\left(T_{j}\right), \delta\left(T_{j}\right) ; T_{j}\right]\right\}=\operatorname{Ln}\left[S\left(T_{j}\right)\right]$.

Dado lo anterior, obtenemos las derivadas parciales para dicha expresión.

$$
\begin{gathered}
\frac{\partial F\left[t, S(t), \delta(t) ; T_{j}\right]}{\partial S(t)}=e^{B\left(T_{j}-t\right) \delta(t)+A\left(T_{j}-t\right)} \\
\frac{\partial^{2} F\left[t, S(t), \delta(t) ; T_{j}\right]}{\partial S(t)^{2}}=0 \\
\frac{\partial F\left[t, S(t), \delta(t) ; T_{j}\right]}{\partial \delta(t)}=S(t) e^{B\left(T_{j}-t\right) \delta(t)+A\left(T_{j}-t\right)} B\left(T_{j}-t\right) \\
\frac{\partial^{2} F\left[t, S(t), \delta(t) ; T_{j}\right]}{\partial \delta(t)^{2}}=S(t)\left[B\left(T_{j}-t\right)\right]^{2} e^{B\left(T_{j}-t\right) \delta(t)+A\left(T_{j}-t\right)} \\
\frac{\partial^{2} F\left[t, S(t), \delta(t) ; T_{j}\right]}{\partial S(t) \partial \delta(t)}=e^{B\left(T_{j}-t\right) \delta(t)+A\left(T_{j}-t\right)} B\left(T_{j}-t\right) \\
\frac{\partial F\left[t, S(t), \delta(t) ; T_{j}\right]}{\partial\left(T_{j}-t\right)}=S(t) e^{B\left(T_{j}-t\right) \delta(t)+A\left(T_{j}-t\right)}\left[\frac{d B\left(T_{j}-t\right)}{d\left(T_{j}-t\right)} \delta(t)+\frac{d A\left(T_{j}-t\right)}{d\left(T_{j}-t\right)}\right]
\end{gathered}
$$

Finalmente, introducimos las distintas derivadas en la ecuación diferencial parcial y simplificamos:

$$
[(r+c)-\delta(t)] S(t)\left\{e^{B\left(T_{j}-t\right) \delta(t)+A\left(T_{j}-t\right)}\right\}
$$




$$
\begin{gathered}
+\{k[\alpha-\delta(t)]-\lambda\}\left\{S(t) e^{B\left(T_{j}-t\right) \delta(t)+A\left(T_{j}-t\right)} B\left(T_{j}-t\right)\right\} \\
+\frac{1}{2} \sigma_{2}^{2} \delta(t) S(t)^{2}\{0\}+\frac{1}{2} \sigma_{2} \delta(t)^{2}\left\{S(t)\left[B\left(T_{j}-t\right)\right]^{2} e^{B\left(T_{j}-t\right) \delta(t)+A\left(T_{j}-t\right)}\right\} \\
+\rho \sigma_{1} \sigma_{2} S(t) \delta(t)\left\{e^{B\left(T_{j}-t\right) \delta(t)+A\left(T_{j}-t\right)} B\left(T_{j}-t\right)\right\} \\
-S(t) e^{B\left(T_{j}-t\right) \delta(t)+A\left(T_{j}-t\right)}\left[\frac{d B\left(T_{j}-t\right)}{d\left(T_{j}-t\right)} \delta(t)+\frac{d A\left(T_{j}-t\right)}{d\left(T_{j}-t\right)}\right]=0 \\
{[(r+c)-\delta(t)]+\{k[\alpha-\delta(t)]-\lambda\} B\left(T_{j}-t\right)+\frac{1}{2} \sigma_{2}^{2} \delta(t)\left[B\left(T_{j}-t\right)\right]^{2}} \\
+\rho \sigma_{1} \sigma_{2} \delta(t) B\left(T_{j}-t\right)=\frac{d B\left(T_{j}-t\right)}{d\left(T_{j}-t\right)} \delta(t)+\frac{d A\left(T_{j}-t\right)}{d\left(T_{j}-t\right)}
\end{gathered}
$$

Esta ecuación se resuelve reduciéndola en dos ecuaciones diferenciales ordinarias de primer orden:

$$
\begin{gathered}
-\delta(t)-k \delta(t) B\left(T_{j}-t\right)+\frac{1}{2} \sigma_{2}{ }^{2} \delta(t)\left[B\left(T_{j}-t\right)\right]^{2}+\rho \sigma_{1} \sigma_{2} \delta(t) B\left(T_{j}-t\right) \\
=\frac{d B\left(T_{j}-t\right)}{d\left(T_{j}-t\right)} \delta(t) \\
(r+c)+k \alpha B\left(T_{j}-t\right)-\lambda B\left(T_{j}-t\right)=\frac{d A\left(T_{j}-t\right)}{d\left(T_{j}-t\right)}
\end{gathered}
$$

Al hallar $A\left(T_{j}-t\right)$ y $B\left(T_{j}-t\right)$ por métodos estándar se tiene la solución a la ecuación diferencial parcial. 